\title{
DYSENTERIC ULCERS IN THE RECTUM WITH GANGRENOUS ULCERS OF THE STOMACH.
}

\author{
By JAMES LITTLE, M.D.; \\ President of the King and Queen's College of Physicians in Ireland; \\ Physician to the Adelaide Hospital.
}

[Read in the Section of Pathology, December 2, 1887.]

IN the middle of last October a man, forty-five years of age, sought admission into the Adelaide Hospital, suffering from severe abdominal pain. It was ascertained from independent sources that he had been of very intemperate habits. He said he had had a cough for many months, and a physical examination showed that he was suffering from phthisis, but not in an advanced stage. In the summer an abscess had formed near the rectum, in consequence of which he was for four or five weeks in the Richmond Asylum, and he left it with a fistulous orifice discharging near the rectum. He remained at home for several weeks in broken down health, and suffering from irritation at the lower end of the rectum. Seven days before he came into the hospital he was, while sitting at the fire, seized with severe pain in the right side of the abdomen, apparently in the direction of the hepatic flexure of the colon. He remained at home suffering from the pain, and from that time until his admission to hospital his bowels only acted once. At the time of admission he complained of pain in the epigastrium, which was swollen and so exquisitely tender that he was unwilling to be examined. After some days the severe pain subsided, but his bowels, which during the first few days that he was in hospital had remained confined, became, without anything having been done to cause such a change, very loose, and the looseness continued from that time until his death. On account of the great discharge of purulent matter there was difficulty in examining his 
stools. He was very unwilling to be moved or disturbed, and I did not see any stools that had a dysenteric character. He died a fortnight after his admission. A post mortem was made by Dr. Bewley, who found small cavities in the lungs. The liver was very large, and had the appearance seen in persons who have died of acute fever. The under-surface of the left lobe of the liver was adherent to the anterior wall of the stomach, and it suddenly gave way and a quantity of purulent matter escaped. A large sloughy-looking ulcer was found in the anterior wall of the stomach, the roof of which was formed by the lower portion of the left lobe of the liver. There was also a small ulcer in the stomach. The sloughy-looking mass connected with the large ulcer was like the surface of a large anthrax, and it communicated with the stomach by an orifice which can be seen in the specimen. The stomach ulcer was not a chronic one, for there was no thickening of the edges, and the surface of the ulcer was covered with gangrenous-looking material. The cæcum was found to be rather extensively ulcerated. It contained several ulcers, but they were rather superficial. In the transverse and descending colons and at the sigmoid flexure ulcers were also numerous. The maximum amount of change was in the rectum and in the cæcum. The question was, had the man suffered from dysentery, and were the ulcers, and other conditions of the stomach, the result of superficial abscesses of the liver opening into the stomach. It was evident that something must have suddenly happened at the time he was seized with the pain, and no doubt at that time the mischief in the liver and stomach first began.a

a It was subsequently ascertained that this patient had been for some weeks during the Summer an inmate of the Richmond Asylum, where dysentery prevailed. 